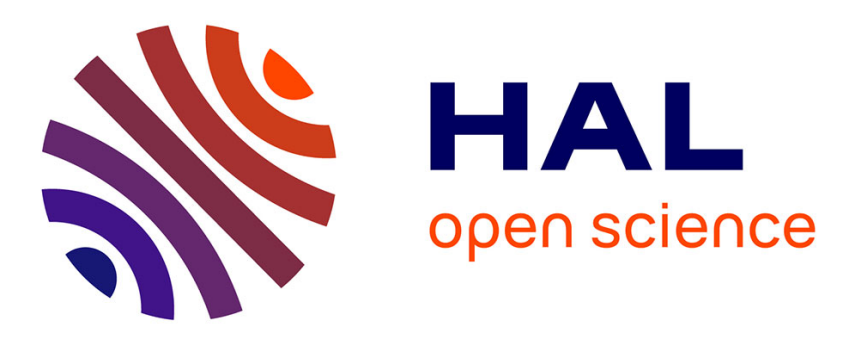

\title{
Filtering and fault estimation of descriptor switched systems
}

Damien Koenig, Benoît Marx, Sébastien Varrier

\section{To cite this version:}

Damien Koenig, Benoît Marx, Sébastien Varrier. Filtering and fault estimation of descriptor switched systems. Automatica, 2016, 63, pp.116-121. 10.1016/j.automatica.2015.10.017 . hal-01227605

\section{HAL Id: hal-01227605 \\ https://hal.science/hal-01227605}

Submitted on 17 Apr 2019

HAL is a multi-disciplinary open access archive for the deposit and dissemination of scientific research documents, whether they are published or not. The documents may come from teaching and research institutions in France or abroad, or from public or private research centers.
L'archive ouverte pluridisciplinaire HAL, est destinée au dépôt et à la diffusion de documents scientifiques de niveau recherche, publiés ou non, émanant des établissements d'enseignement et de recherche français ou étrangers, des laboratoires publics ou privés. 


\title{
Filtering and fault estimation of descriptor switched systems
}

\author{
Damien Koenig ${ }^{\mathrm{a}}$, Benoît Marx ${ }^{\mathrm{b}, \mathrm{c}, *}$, Sébastien Varrier $^{\mathrm{a}}$ \\ ${ }^{a}$ Gipsa-Lab, 11 rue des Mathématiques, Grenoble Campus BP 46, 38402 Saint Martin d'Hères Cedex, France \\ ${ }^{b}$ Université de Lorraine, CRAN, UMR 7039, 2 avenue de la Foret de Haye, 54516 Vandoeuvre-les-Nancy, France \\ ${ }^{c}$ CNRS, CRAN, UMR 7039, France
}

\begin{abstract}
In this paper, the problems of state and fault estimation are addressed for a class of switched descriptor systems subject to Lipschitz nonlinearities and unknown inputs (UI). The UI appear both on the dynamic and on the measurement equations. Two problems are addressed by $\mathcal{L}_{2}$-gain minimization with the use of switched Lyapunov functions and formulated by LMI. First, a functional observer for switched Lipschitz nonlinear descriptor system is proposed for robust state estimation. Second, fault estimation is performed by filtering the output estimation error, as usually done in the residual generation framework. Moreover, frequency weighting functions can be used to shape the response to the fault and thus improve the estimation.
\end{abstract}

Keywords: Descriptor systems, nonlinear switched systems, fault estimation, $H_{\infty}$ functional observer.

\section{Introduction}

Controller and/or observer design for switched systems has recently received much attention. Switched systems are a class of hybrid systems defined by a collection of dynamical (linear and/or nonlinear) subsystems together with a switching rule specifying the switching between the subsystems. Surveys on switched systems are available in [15, 21, 5]. Many arising problems were treated for switched systems: stability [11, 20], output-feedback [6, 8], state estimation [14].

The descriptor systems generalize the state-space systems by encompassing both differential and static relations [16, 7, 22]. Many results have been extended to descriptor systems concerning stability, control or state estimation [7, 19], fault estimation [12] and fault tolerant control [9, 18].

The motivation of the present work is to extend some results on state and fault estimation to switched descriptor systems subjected to unknown inputs (UI), faults and Lipschitz nonlinearities. This formalism allows to model systems with both dynamic and static behaviors, with functioning mode changes and which inputs are partially unavailable to measurement (fault, disturbance, etc). Despite its generality, only few results exist for the class of discrete time switched descriptor systems (DTSDS). In [10, 17] stability of Markovian descriptor systems are studied. In [13], $H_{\infty}$-filtering and state feedback are treated but no nonlinearities are considered. Proposing a unified approach to robust state filtering and fault diagnosis for DTSDS, two objectives are aimed here. The first objective is to relax the perfect UI decoupling conditions needed for state estimation in [14] by using the $\mathcal{L}_{2}$-approach. Moreover, in order to obtain relaxed stability conditions, multiple Lyapunov functions

\footnotetext{
${ }^{*}$ Corresponding author

Email addresses: damien.koenig@esisar.grenoble-inp.fr (Damien Koenig), benoit.marx@univ-lorraine.fr (Benoît Marx), sebastien.varrier@esisar.grenoble-inp.fr (Sébastien Varrier)
}

are used to derive LMI conditions and introduce some non necessary positive definite slack variables. The second one is to perform robust fault diagnosis via robust fault estimation and to generalize [14], where only disturbance UI were envisaged, but no diagnosis was performed. The objective of fault diagnosis is to highlight the faults (actuator or sensor dysfunction) while being robust to the disturbance UI [1]. In observer-based fault diagnosis, the output estimation error is usually used as a primary residual signal. This primary residual signal, affected by both disturbance and fault, is filtered by a post-filter to obtain a robust fault estimate. The state and fault estimation are performed in a unified way since the generating systems of their respective error estimations is written similarly. One should note that no previous works have considered the problem of robust fault diagnosis for nonlinear switched descriptor systems.

This note is organized as follows. The problem is stated in section 2 . In section 3 , the state and fault observers are designed. Before concluding, section 4 is devoted to a numerical example.

Notation 1. For any square matrix $M, M>0$ (resp. $M<$ $0)$ means that the matrix $M$ is a real symmetric positive (resp. negative) definite and $\mathbb{S}(M)$ is defined by $\mathbb{S}(M)=M+M^{T}$. The blocks induced by symmetry are denoted $*, I_{n}$ is the $n \times n$ identity matrix, $0_{n}\left(\right.$ resp. $\left.0_{n \times m}\right)$ is the $n \times n($ resp. $n \times m)$ null matrix and diag $\left(X_{1}, \ldots, X_{n}\right)$ is the block diagonal matrix which diagonal entries are $X_{1}, \ldots, X_{n}$. The set of the $N$ first strictly positive integers is denoted $\mathbf{N}_{N}=\{1, \ldots N\}$ and $\ell_{2}[0, \infty)$ denotes the space of square summable infinite vector sequences with the usual norm $\left\|_{.}\right\|_{2}$. 


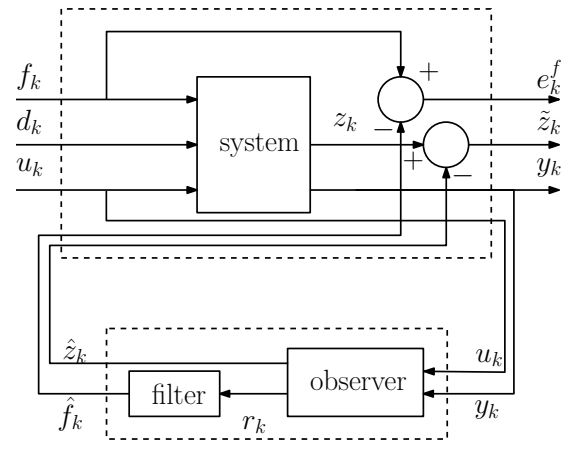

Figure 1: State filtering and fault estimation scheme

\section{Problem formulation}

Consider the following DTSDS

$$
\begin{aligned}
& \sum_{j=1}^{N} \alpha_{j}(k+1) E_{j} x_{k+1}=\sum_{i=1}^{N} \alpha_{i}(k)\left(A_{i} x_{k}+B_{i} u_{k}\right. \\
& \left.\quad+R_{i}^{1} f_{k}+W_{i}^{1} d_{k}+H_{i} \phi\left(x_{k}, u_{k}, k\right)\right) \\
& y_{k}=\sum_{i=1}^{N} \alpha_{i}(k)\left(C_{i} x_{k}+D_{i} u_{k}+R_{i}^{2} f_{k}+W_{i}^{2} d_{k}\right) \\
& z_{k}=\sum_{i=1}^{N} \alpha_{i}(k) T_{i} x_{k}
\end{aligned}
$$

where $x \in \mathbb{R}^{n}, d \in \mathbb{R}^{n_{d}}, f \in \mathbb{R}^{n_{f}}, u \in \mathbb{R}^{n_{u}}, \phi: \mathbb{R}^{n} \times \mathbb{R}^{n_{u}} \times \mathbb{N} \rightarrow$ $R^{n_{\phi}}, y \in \mathbb{R}^{m}$ and $z \in \mathbb{R}^{q}$ denote respectively the state, the UI, the fault, the control input, the Lipschitz nonlinearity, the output vector and the vector to be estimated, with $q \leq n$. The matrices $E_{j}$ may be singular. The functions $\alpha_{i}: \mathbb{N} \rightarrow\{0,1\}\left(i \in \mathbf{N}_{N}\right)$ are the known switching signals satisfying $\sum_{i=1}^{N} \alpha_{i}(k)=1(k \in \mathbb{N})$ and specifying the activated subsystem: $\alpha_{i}(k)=1$ and $\alpha_{j}(k+$ $1)=1$ mean that the matrices $\left(E_{j}, A_{i}, B_{i}, W_{i}, C_{i}\right)$ are activated at time $k$. The measurements $y$, the vector $z$, the fault $f$ and the UI $d$ are respectively assumed to be linearly independent i.e.

$$
\begin{aligned}
& \operatorname{rank}\left(\left[\begin{array}{llll}
C_{i} & D_{i} & R_{i}^{2} & W_{i}^{2}
\end{array}\right]\right)=m \\
& \operatorname{rank}\left(T_{i}\right)=q \\
& \operatorname{rank}\left(\left[\begin{array}{ll}
R_{i}^{1} & W_{i}^{1} \\
R_{i}^{2} & W_{i}^{2}
\end{array}\right]\right)=n_{f}+n_{d}
\end{aligned}
$$

Assumptions: In the sequel it is assumed that:

(A1) the nonlinearity $\phi\left(x_{k}, u_{k}, k\right)$ is globally Lipschitz in $x_{k}$ i.e. there exist a constant $\beta$ s.t. $\forall u_{k} \in \mathbb{R}^{n_{u}}$ and $\forall k \in \mathbb{N}$

$$
\left\|\phi\left(x_{k}, u_{k}, k\right)-\phi\left(\hat{x}_{k}, u_{k}, k\right)\right\| \leq \beta\left\|x_{k}-\hat{x}_{k}\right\|
$$

(A2) for $i \in \mathbf{N}_{N}$, the triplet $\left(E_{i}, A_{i}, C_{i}\right)$ is impulse observable and detectable [7].

Problem 1. Consider the switched functional observer (SFO) for the fault free DTSDS (1)

$$
\begin{gathered}
\sum_{j=1}^{N} \alpha_{j}(k+1) E_{j} \hat{x}_{k+1}=\sum_{i=1}^{N} \alpha_{i}(k)\left(A_{i} \hat{x}_{k}\right. \\
\left.+B_{i} u_{k}+H_{i} \phi\left(\hat{x}_{k}, u_{k}, k\right)+L_{i} r_{k}\right) \\
\hat{y}_{k}=\sum_{i=1}^{N} \alpha_{i}(k)\left(C_{i} \hat{x}_{k}+D_{i} u_{k}\right) \\
\hat{z}_{k}=\sum_{i=1}^{N} \alpha_{i}(k) T_{i} \hat{x}_{k} \\
r_{k}=y_{k}-\hat{y}_{k}
\end{gathered}
$$

where $L_{i}$ are the observer gains, $\hat{x}$ and $\hat{z}$ are the estimate of $x$ and $z$ and $r$ is the output estimation error. The gains $L_{i}$ are determined such that:

$\left(S_{1}\right)$ the state estimation error, $e_{k}=x_{k}-\hat{x}_{k}$, is generated by a globally asymptotically stable and impulse free system, when $d_{k}{ }^{T}=0$ and $f_{k}^{T}=0$;

$\left(S_{2}\right)$ the $\mathcal{L}_{2}$-gain from the UI $d_{k}$ to the estimation error $\tilde{z}_{k}=$ $z_{k}-\hat{z}_{k}$ is bounded by a prescribed positive scalar $\gamma_{1}$.

Problem 2. A post-filter is designed in order to estimate the fault. The proposed SFO and post-filter are defined by (3) and (4) respectively.

$$
\begin{aligned}
x_{k+1}^{F} & =\sum_{i=1}^{N} \alpha_{i}(k)\left(A_{i}^{F} x_{k}^{F}+B_{i}^{F} r_{k}\right) \\
\hat{f}_{k} & =\sum_{i=1}^{N} \alpha_{i}(k)\left(C_{i}^{F} x_{k}^{F}+D_{i}^{F} r_{k}\right)
\end{aligned}
$$

where $\hat{f}_{k}$ is the fault estimate and $x_{k}^{F} \in \mathbb{R}^{n_{F}}$ is the filter state. The problem is then to simultaneously determine the gains $L_{i}$ and the matrices $A_{i}^{F}, B_{i}^{F}, C_{i}^{F}$ and $D_{i}^{F}$ satisfying the following specifications.

$\left(S_{3}\right)$ the state and fault estimation errors $\left(e_{k}=x_{k}-\hat{x}_{k}\right.$ and $\left.e_{k}^{f}=f_{k}-\hat{f}_{k}\right)$ are generated by a globally asymptotically stable and impulse free system, when $d_{k}=0$ and $f_{k}=0$;

$\left(S_{4}\right)$ the $\mathcal{L}_{2}$-gain from $\left(w_{k}\right)^{T}=\left[\left(d_{k}\right)^{T}\left(f_{k}\right)^{T}\right]$ to the fault estimation error $e_{k}^{f}$ is bounded by a prescribed positive scalar $\gamma_{2}$.

This design procedure can be viewed as a standard $H_{\infty}$-control problem, as shown in figure 1.

\section{Robust functional observer design and fault diagnosis}

The gains of the SFO (3) and filter (4) for the DTSDS (1) are obtained by solving an LMI problem. Two Lyapunov candidate functions are considered :

$$
\begin{aligned}
V\left(e_{k}\right) & =\sum_{i=1}^{N} \alpha_{i}(k) e_{k}^{T} E_{i}^{T} P_{i} E_{i} e_{k} \\
V^{a}\left(e_{k}^{a}\right) & =\sum_{i=1}^{N} \alpha_{i}(k) e_{k}^{a T} E_{i}^{a T} P_{a_{i}} E_{i}^{a} e_{k}^{a}
\end{aligned}
$$


where $P_{i}$ and $P_{a_{i}}$ are symmetric matrices and where $\left(e_{k}^{a}\right)^{T}=$ $\left[\left(e_{k}\right)^{T}\left(x_{k}^{F}\right)^{T}\left(e_{k}^{f}\right)^{T}\right]$. It is well-known [2,4] that for such Lyapunov functions:

- $\Delta V\left(e_{k}\right)=V\left(e_{k+1}\right)-V\left(e_{k}\right)<0$ implies $\left(S_{1}\right)$

- $H_{e_{k}}\left(\gamma_{1}\right)=\Delta V\left(e_{k}\right)+\tilde{z}_{k}^{T} \tilde{z}_{k}-\gamma_{1}^{2} d_{k}^{T} d_{k}<0$ implies $\left(S_{2}\right)$

- $\Delta V^{a}\left(e_{k}^{a}\right)=V^{a}\left(e_{k+1}^{a}\right)-V^{a}\left(e_{k}^{a}\right)<0$ implies $\left(S_{3}\right)$

- $H_{e_{k}^{a}}\left(\gamma_{2}\right)=\Delta V^{a}\left(e_{k}^{a}\right)+e_{k}^{f^{T}} e_{k}^{f}-\gamma_{2}^{2} w_{k}^{T} w_{k}<0$ implies $\left(S_{4}\right)$

\subsection{State filtering in the fault free case}

The first objective is to develop a SFO (3) for the fault free DTSDS (1), such that the specifications $\left(S_{1}\right)$ and $\left(S_{2}\right)$ are fulfilled. The filtering error between (3) and (1) is generated by

$$
\begin{aligned}
\sum_{j=1}^{N} \alpha_{j}(k+1) E_{j} e_{k+1} & =\sum_{i=1}^{N} \alpha_{i}(k)\left(A_{c l_{i}} e_{k}\right. \\
& \left.+H_{i} \tilde{\phi}_{k}+W_{c l_{i}} d_{k}\right) \\
\tilde{z}_{k} & =\sum_{i=1}^{N} \alpha_{i}(k) T_{i} e_{k}
\end{aligned}
$$

where $\tilde{\phi}_{k}=\phi\left(x_{k}, u_{k}, k\right)-\phi\left(\hat{x}_{k}, u_{k}, k\right), A_{c l_{i}}=\left(A_{i}-L_{i} C_{i}\right)$ and $W_{c l_{i}}=\left(W_{i}^{1}-L_{i} W_{i}^{2}\right)$.

The following result details sufficient LMI existence conditions of the SFO and the computation of the gains $L_{i}$.

Theorem 1. The SFO (3) for the DTSDS (1), satisfying ( $S 1)$ and $(S 2)$ exists if the triplets $\left(E_{i}, A_{i}, C_{i}\right)$ are finite dynamics detectable and impulse observable (see [13] for conditions) and is obtained by finding the symmetric matrices $P_{i} \in \mathbb{R}^{n \times n}$, symmetric positive definite matrices $G_{i} \in \mathbb{R}^{n \times n}$, matrices $\tilde{G}_{i} \in \mathbb{R}^{n \times m}$ and $M_{i} \in \mathbb{R}^{\left(n+n_{d}+n_{\phi}\right) \times n}$ minimizing $\bar{\gamma}_{1}$ under the constraints (8a8c) for $(i, j) \in \mathbf{N}_{N}^{2}$.

$$
\begin{aligned}
E_{i}^{T} P_{i} E_{i} & \geq 0 \\
\mathcal{M}_{i j i} & <0 \\
\mathcal{M}_{i j j} & <0
\end{aligned}
$$

where $\bar{\gamma}_{1}=\gamma_{1}^{2}$ and

$$
\begin{aligned}
\mathcal{M}_{i j k}= & {\left[\begin{array}{cccc}
\Theta_{i k} & \bar{\Theta}_{i} & M_{i} & \tilde{C}_{i}^{T} \tilde{G}_{i}^{T} \\
* & P_{j}-2 G_{i} & 0 & 0 \\
* & * & -G_{i} & 0 \\
* & * & -G_{i}
\end{array}\right] } \\
\bar{\Theta}_{i}= & \left(\tilde{A}_{i}^{T} G_{i}^{T}-\tilde{C}_{i}^{T} \tilde{G}_{i}^{T}-M_{i}\right) \\
\Theta_{i k}= & \tilde{T}_{i k}+\tilde{C}_{i}^{T} C_{i} G_{i} C_{i}^{T} \tilde{C}_{i} \\
& +\mathbb{S}\left(M_{i} \tilde{A}_{i}+M_{i} C_{i}^{T} \tilde{C}_{i}+\tilde{C}_{i}^{T} C_{i} \tilde{G}_{i} \tilde{C}_{i}\right) \\
\tilde{T}_{i k}= & \operatorname{diag}\left(\beta^{2} I_{n}+T_{i}^{T} T_{i}-E_{k}{ }^{T} P_{i} E_{k},-\bar{\gamma}_{1} I_{n_{d}},-I_{n_{\phi}}\right) \\
\tilde{A}_{i}= & {\left[\begin{array}{lll}
A_{i} & W_{i}^{1} & H_{i}
\end{array}\right] } \\
\tilde{C}_{i}= & {\left[\begin{array}{lll}
C_{i} & W_{i}^{2} & 0
\end{array}\right] }
\end{aligned}
$$

The observer gains are obtained by

$$
L_{i}=G_{i}^{-1} \tilde{G}_{i}
$$

Proof. The disturbance attenuation of the DTSDS (7) expressed by $S_{2}$ has to be satisfied under arbitrary switching laws, it follows that $H_{e_{k}}\left(\gamma_{1}\right)<0$ and (7) are respectively equivalent to

$$
\begin{aligned}
& e_{k+1}{ }^{T} E_{j}^{T} P_{j} E_{j} e_{k+1}-\gamma_{1}{ }^{2} d_{k}^{T} d_{k} \\
& \quad-e_{k}^{T}\left(E_{i}^{T} P_{i} E_{i}-T_{i}^{T} T_{i}\right) e_{k}<0
\end{aligned}
$$

and

$$
\begin{aligned}
E_{j} e_{k+1} & =A_{c l_{i}} e_{k}+H_{i} \tilde{\phi}_{k}+W_{c l_{i}} d_{k} \\
\tilde{z}_{k} & =T_{i} e_{k}
\end{aligned}
$$

where $i$ (resp. $j$ ) is the number of the activated model at time $k$ (resp. $k+1$ ). Substituting (11) into (10), the following inequality is obtained

$$
\begin{aligned}
H_{e_{k}}\left(\gamma_{1}\right)=(*) & P_{j}\left[A_{c l_{i}} e_{k}+H_{i} \tilde{\phi}_{k}+W_{c l_{i}} d_{k}\right] \\
& +e_{k}^{T}\left(T_{i}^{T} T_{i}-E_{i}^{T} P_{i} E_{i}\right) e_{k}-\gamma_{1}{ }^{2} d_{k}{ }^{T} d_{k}<0
\end{aligned}
$$

Defining $\tilde{\Theta}_{i j}=\Theta_{i j}+M_{i} G_{i}^{-1} M_{i}{ }^{T}+\tilde{C}_{i}{ }^{T} \tilde{G}_{i}{ }^{T} G_{i}^{-1} \tilde{G}_{i} \tilde{C}_{i}$, if (8b) hold and with two Schur complements, $(8 \mathrm{~b})$ is equivalent to

$$
\left[\begin{array}{cc}
\tilde{\Theta}_{i i} & \tilde{A}_{i}^{T} G_{i}^{T}-\tilde{C}_{i}^{T} \tilde{G}_{i}^{T}-M_{i} \\
* & P_{j}-2 G_{i}
\end{array}\right]<0
$$

From (8b), the matrices $G_{i}>0$ and defining $\tilde{M}_{i}=M_{i}{ }^{T}+\tilde{G}_{i} \tilde{C}_{i}+$ $G_{i} C_{i}{ }^{T} \tilde{C}_{i}$, it follows $\tilde{M}_{i}^{T} G_{i}^{-1} \tilde{M}_{i} \geq 0$ or equivalently

$$
\begin{aligned}
&-\mathbb{S}\left(M_{i} G_{i}^{-1} \tilde{G}_{i} \tilde{C}_{i}\right) \leq M_{i} G_{i}^{-1} M_{i}{ }^{T} \\
&+\tilde{C}_{i}{ }^{T} \tilde{G}_{i}{ }^{T} G_{i}^{-1} \tilde{G}_{i} \tilde{C}_{i}+\tilde{C}_{i}{ }^{T} C_{i} G_{i} C_{i}{ }^{T} \tilde{C}_{i} \\
& \quad+\mathbb{S}\left(M_{i} C_{i}{ }^{T} \tilde{C}_{i}+\tilde{C}_{i}{ }^{T} C_{i} \tilde{G}_{i} \tilde{C}_{i}\right)
\end{aligned}
$$

Adding $\tilde{T}_{i i}+\mathbb{S}\left(M_{i} \tilde{A}_{i}\right)$ to both sides of (14), it follows

$$
\tilde{T}_{i i}+\mathbb{S}\left(M_{i} \tilde{A}_{i}-M_{i} G_{i}^{-1} \tilde{G}_{i} \tilde{C}_{i}\right) \leq \tilde{\Theta}_{i j}<0
$$

From (15), with (9) and $\tilde{A}_{c l i}=\tilde{A}_{i}-L_{i} \tilde{C}_{i}$, it follows that (13) implies

$$
\left[\begin{array}{cc}
\tilde{T}_{i i}+\mathbb{S}\left(M_{i} \tilde{A}_{c l i}\right) & \tilde{A}_{c l i}^{T} G_{i}^{T}-M_{i} \\
* & P_{j}-2 G_{i}
\end{array}\right]<0
$$

Pre- and post-multiplying (16) by $\left[I \tilde{A}_{c l i}^{T}\right]$ and its transpose, it follows that (16) is equivalent to

$$
\tilde{T}_{i i}+\tilde{A}_{c l i}^{T} P_{j} \tilde{A}_{c l i}<0
$$

Pre- and post-multiplying (17) by $\left[e_{k}^{T} d_{k}^{T} \tilde{\phi}_{k}^{T}\right]$ and its transpose, (17) becomes

$$
H_{e_{k}}\left(\gamma_{1}\right)+\beta^{2} e_{k}^{T} e_{k}-\tilde{\phi}_{k}^{T} \tilde{\phi}_{k}<0
$$

where $H_{e_{k}}\left(\gamma_{1}\right)$ is defined by (12). Since the nonlinearity is assumed to be Lipschitz in $x$ (2), then

$$
\beta^{2} e_{k}^{T} e_{k}-\tilde{\phi}_{k}^{T} \tilde{\phi}_{k} \geq 0
$$


From (18) and (19) it follows that $H_{e_{k}}\left(\gamma_{1}\right)<0$ and thus $\left(S_{2}\right)$ is satisfied. Moreover, when $d_{k}=0$ in $H_{e_{k}}\left(\gamma_{1}\right)$, it obviously follows that $\Delta V\left(e_{k}\right)<0$. From definition 1.1 of [4] the system (11) is stable. Following the same steps, (8c) implies $\tilde{T}_{i j}+\tilde{A}_{c l i}^{T} P_{j} \tilde{A}_{c l i}<0$. Pre- and post multiplying this inequality by $\left[I_{n} 0_{n_{d}} 0_{n_{\Phi}}\right]$ and its transpose, one obtains $\left(A_{i}-L_{i} C_{i}\right)^{T} P_{j}\left(A_{i}-\right.$ $\left.L_{i} C_{i}\right)-E_{j}^{T} P_{i} E_{j}<0$ for $(i, j) \in \mathbf{N}_{N}^{2}$, implying that (11) is impulse free. Thus $\left(S_{1}\right)$ holds which achieves the proof.

Remark 1. The objective is the minimization of the $\mathcal{L}_{2}$-gain from $d_{k}$ to $e_{k}$, consequently the perfect decoupling is not sought and the conditions assumed in [14] are not needed to solve the LMIs (8).

The special case when the system is neither affected by disturbances nor by nonlinearities (i.e. $f_{k}=0, d_{k}=0$ and $\left.\phi\left(x_{k}, u_{k}, k\right)=0\right)$ is briefly envisaged in the following corollary.

Corollary 1. A SFO (3) for the DTSDS (1) with $f_{k}=d_{k}=$ $\phi\left(x_{k}, u_{k}, k\right)=0$ exists and satisfies $\left(S_{1}\right)$ if there exist symmetric matrices $P_{i} \in \mathbb{R}^{n \times n}$, matrices $U_{i} \in \mathbb{R}^{n \times n}$ and $M_{i} \in \mathbb{R}^{m \times n}$ satisfying (8) for $(i, j) \in \mathbf{N}_{N}^{2}$ with $\mathcal{M}_{i j k}$ defined by

$$
\begin{aligned}
\mathcal{M}_{i j k} & =\left[\begin{array}{cc}
\bar{\Theta}_{i k} & * \\
-U_{i}^{T}+U_{i} A_{i}-M_{i} C_{i} & P_{j}-\mathbb{S}\left(U_{i}\right)
\end{array}\right] \\
\bar{\Theta}_{i k} & =\mathbb{S}\left(U_{i} A_{i}-M_{i} C_{i}\right)-E_{k}{ }^{T} P_{i} E_{k}
\end{aligned}
$$

The observer gains are: $H_{i}=0$ and $L_{i}=U_{i}^{-1} M_{i}$.

Proof. Consider (8b) defined with (20) and $U_{i} L_{i}=M_{i}$, preand post-multiplying it by $\left[I_{n} A_{c l i}^{T}\right]$ and its transpose, then $A_{c l i}{ }^{T} P_{j} A_{c l i}-E_{i}{ }^{T} P_{i} E_{i}<0$ and consequently $\Delta V\left(e_{k}\right)<0$ follows. From (8c), impulse freeness is obtained like in the proof of theorem 1 and $\left(S_{1}\right)$ follows.

Remark 2. For $A_{c l_{i}}=A, E_{i}=E$ and $P_{i}=P_{j}=P$, the inequalities (8) with (20) imply the LMIs defined in lemma 1 of [23]. For $A_{c l_{i}}=A_{i}$ and $E_{i}=E$, they are equivalent to the LMIs defined in [22]. For $E_{i}=E$ and by the duality principle, the results of corollary 1 coincides with the results of Theorem 1 of [3]. Thus, corollary 1 can be considered as a generalization of these works.

\subsection{Fault estimation}

In order to simultaneously design the SFO (3) and the filter (4), the DTSDS generating the state and fault estimation errors is written as

$$
\begin{gathered}
\sum_{j=1}^{N} \alpha_{j}(k+1) E_{j}^{a} e_{k+1}^{a}=\sum_{i=1}^{N} \alpha_{i}(k)\left(A_{c l i}^{a} e_{k}^{a}\right. \\
\left.+W_{c l i}^{a} w_{k}+H_{i}^{a} \tilde{\phi}_{k}\right) \\
e_{k}^{f}=\sum_{i=1}^{N} \alpha_{i}(k) T_{i}^{a} e_{k}^{a}
\end{gathered}
$$

where $e_{k}^{a T}=\left[\begin{array}{lll}e_{k}^{T} & x_{k}^{F^{T}} & e_{k}^{f^{T}}\end{array}\right], A_{c l i}^{a}=A_{i}^{a}-L_{i}^{a} C_{i}^{a}, W_{c l i}^{a}=W_{i}^{a 1}-$ $L_{i}^{a} W_{i}^{a 2}, E_{j}^{a}=\operatorname{diag}\left(E_{j}, I_{n_{F}}, 0_{n_{f}}\right)$ and

$$
\begin{aligned}
A_{i}^{a} & =\left[\begin{array}{ccc}
A_{i} & 0 & 0 \\
0 & 0 & 0 \\
0 & 0 & -I_{n_{f}}
\end{array}\right] \quad L_{i}^{a}=\left[\begin{array}{cc}
L_{i} & 0 \\
-B_{i}^{F} & -A_{i}^{F} \\
D_{i}^{F} & C_{i}^{F}
\end{array}\right] \\
W_{i}^{a 1} & =\left[\begin{array}{cc}
W_{i}^{1} & R_{i}^{1} \\
0 & 0 \\
0 & I_{n_{f}}
\end{array}\right] \quad H_{i}^{a}=\left[\begin{array}{c}
H_{i} \\
0 \\
0
\end{array}\right] \quad T_{i}^{a T}=\left[\begin{array}{c}
0 \\
0 \\
I_{n_{f}}
\end{array}\right] \\
C_{i}^{a} & =\left[\begin{array}{ccc}
C_{i} & 0 & 0 \\
0 & I_{n_{F}} & 0
\end{array}\right] \quad W_{i}^{a 2}=\left[\begin{array}{cc}
W_{i}^{2} & R_{i}^{2} \\
0 & 0
\end{array}\right]
\end{aligned}
$$

Since (21) is similar to (7) up to matrix and state vector augmentations, the theorem 1 can be adapted to determine the observer and post filter gains $L_{i}^{a}$ such that (21) satisfies $\left(S_{3}\right)$ and $\left(S_{4}\right)$.

Corollary 2. The SFO (3) and post-filter (4) for the DTSDS (1), satisfying $(S 3)$ and $(S 4)$ exist if the triplets $\left(E_{i}, A_{i}, C_{i}\right)$ are finite dynamics detectable and impulse observable (see [13] for conditions) and are obtained by finding the symmetric matrices $P_{i} \in \mathbb{R}^{\left(n+n_{F}+n_{f}\right) \times\left(n+n_{F}+n_{f}\right)}$, symmetric positive definite matrices $G_{i} \in \mathbb{R}^{\left(n+n_{F}+n_{f}\right) \times\left(n+n_{F}+n_{f}\right)}$, matrices $\tilde{G}_{i} \in \mathbb{R}^{\left(n+n_{F}+n_{f}\right) \times\left(m+n_{F}\right)}$ and $M_{i} \in \mathbb{R}^{\left(n+n_{F}+2 n_{f}+n_{d}+n_{\phi}\right) \times\left(n+n_{F}+n_{f}\right)}$ minimizing $\bar{\gamma}_{2}$ under the constraints (8b-8c-22) for $(i, j) \in \mathbf{N}_{N}^{2}$.

$$
E_{i}^{a T} P_{i} E_{i}^{a} \geq 0
$$

where $\bar{\gamma}_{2}=\gamma_{2}^{2}$ and

$$
\begin{aligned}
\Theta_{i k} & =\tilde{T}_{i k}^{a}+\tilde{C}_{i}^{a T} C_{i}^{a} G_{i} C_{i}^{a T} \tilde{C}_{i}^{a} \\
& +\mathbb{S}\left(M_{i} \tilde{A}_{i}^{a}+M_{i} C_{i}^{a T} \tilde{C}_{i}^{a}+\tilde{C}_{i}^{a T} C_{i}^{a} \tilde{G}_{i} \tilde{C}_{i}^{a}\right) \\
\tilde{T}_{i k} & =\operatorname{diag}\left(\operatorname{diag}\left(\beta^{2} I_{n}, 0_{n_{F}+n_{f}}\right)\right. \\
& \left.+T_{i}^{a T} T_{i}^{a}-E_{k}^{a T} P_{i} E_{k}^{a},-\bar{\gamma}_{2} I_{n_{d}+n_{f}},-I_{n_{\phi}}\right) \\
\tilde{A}_{i} & =\left[\begin{array}{lll}
A_{i}^{a} & W_{i}^{a 1} & H_{i}^{a}
\end{array}\right] \\
\tilde{C}_{i} & =\left[\begin{array}{lll}
C_{i}^{a} & W_{i}^{a 2} & 0
\end{array}\right]
\end{aligned}
$$

The observer and filter gains are given by $L_{i}^{a}=G_{i}^{-1} \tilde{G}_{i}$, where $G_{i}=\operatorname{diag}\left(G_{i}^{1}, G_{i}^{2}\right)$ and

$$
\tilde{G}_{i}=\left[\begin{array}{cc}
\tilde{G}_{i}^{1} & 0_{n \times n_{F}} \\
\tilde{G}_{i}^{2} & \tilde{G}_{i}^{3} \\
\tilde{G}_{i}^{4} & \tilde{G}_{i}^{5}
\end{array}\right]
$$

with $G_{i}^{1} \in \mathbb{R}^{n \times n}$ and $G_{i}^{2} \in \mathbb{R}^{\left(n_{F}+n_{f}\right) \times\left(n_{F}+n_{f}\right)}$.

Proof. The proof is similar to the one of theorem 1 and thus omitted.

Remark 3. The null block in $\tilde{G}_{i}$ does not introduce any equality constraint, since it suffices to use the secondary LMI variables $\tilde{G}_{i}^{1}, \ldots, \tilde{G}_{i}^{5}$ in (23). The null block of $\tilde{G}_{i}$ and the block diagonal structure of $G_{i}$ imply the nullity of the $(1,2)$ block of $L_{i}^{a}$. 


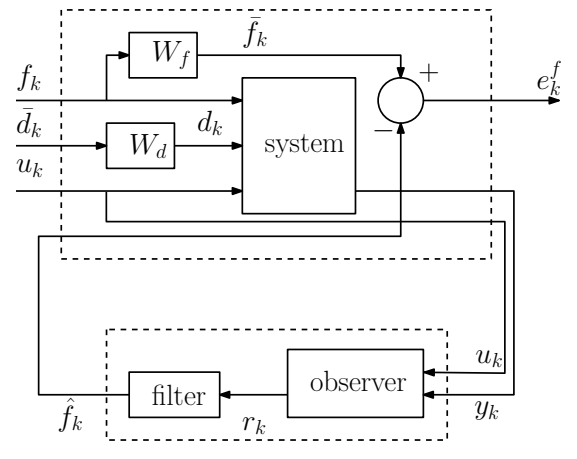

Figure 2: Improved robust fault diagnosis scheme

Remark 4. If the main purpose is state estimation or filtering rather than fault estimation, the estimator design should be slightly modified by adding $R_{i}^{1} \hat{f}_{k}$ in (3a), $R_{i}^{2} \hat{f}_{k}$ and (3b) and by minimizing the $\mathcal{L}_{2}$-gain from the exogenous inputs to the estimation or filtering error e or $\tilde{z}$.

Remark 5. Analogously to standard $H_{\infty}$-control and as depicted by figure 2, dynamical filters can be introduced in the design procedure in order to improve the fault diagnosis and avoid to impose hard constraints on the whole frequency range (see chapter 6.5 of [1]). The filter $W_{d}$ imposes an attenuation level of the UI in specific frequency ranges and $W_{f}$ is introduced to define the desired frequency response of $\hat{f}_{k}$ to the fault.

\section{Numerical example}

The following example illustrates the performance of the SFO (3) and the filter (4), proposed in section 3. Consider the DTSDS (1) defined by $\phi\left(x_{k}, u_{k}\right)=0.1 \sin \left(x_{2 k}\right), E_{i}=\operatorname{diag}(0,1)$ and

$$
\begin{gathered}
A_{1}=\left(\begin{array}{cc}
0.9 & 0.1 \\
0 & 0.1
\end{array}\right), A_{2}=\left(\begin{array}{cc}
0.7 & 0.15 \\
0 & 0.1
\end{array}\right), \\
A_{3}=\left(\begin{array}{cc}
0.5 & 0.15 \\
0 & 0.1
\end{array}\right), B_{i}=\left(\begin{array}{l}
i \\
0
\end{array}\right), H_{i}=\left(\begin{array}{l}
1 \\
0
\end{array}\right), \\
R_{i}^{1}=\left(\begin{array}{l}
1 \\
0
\end{array}\right), W_{i}^{1}=\left(\begin{array}{c}
0 \\
0.1
\end{array}\right), C_{i}^{T}=\left(\begin{array}{c}
1 \\
0.1
\end{array}\right), T_{i}^{T}=\left(\begin{array}{l}
0 \\
1
\end{array}\right)
\end{gathered}
$$

$D_{i}=0, R_{i}^{2}=0$ and $W_{i}^{2}=0.3$, for $i \in \mathbf{N}_{3}$. The fault $f_{k}$ affects the first system state and the perturbation $d_{k}$ affects the second state and the measurement output $y_{k}$. One can readily verify that the system does not satisfy the UI decoupling conditions of [14]. The considered perturbation is a white noise. The system inputs are presented in figures 3(a) and 3(b). The estimation results, obtained for $\hat{x}_{0}=\left[\begin{array}{ll}1 & 1\end{array}\right]^{T}$, are presented in figures 3(c) and 3(d). One can notice that the noise disturbance is well attenuated, and the estimator fast converges to the good value. The filter proposed in (4) is implemented in order to estimate the fault $f_{k}$. The result of the fault estimation is presented in figure 3(e). According to the remark 5, lowpass filters $W_{f}$ and $W_{d}^{-1}$ are used and the improved results presented in figure 3(f) are obtained. One can note that the estimation of the faults quickly converge toward the real value of the fault, despite the nonlinearities and the noise.

\section{Conclusion}

In this paper, a robust state and fault observer is designed for discrete-time switched nonlinear descriptor systems. This generic class of systems were not envisaged in the diagnosis framework. The design objectives are to minimize the $\mathcal{L}_{2}$-gain from the unknown inputs to the state and fault estimation errors. LMI conditions are obtained using switched Lyapunov functions to avoid conservatism introduced by single Lyapunov functions and filters can be introduced to improve the fault estimation robustness. The proposed approach could be extended to a wide class of systems such as LPV or descriptor TakagiSugeno systems.

[1] Blanke, M., Kinnaert, M., Lunze, J., Staroswiecki, M., 2006. Diagnosis and Fault-Tolerant Control. Springer.

[2] Boyd, S., Ghaoui, L.E., Feron, E., Balakrishnan, V., 1994. Linear Matrix Inequalities in Systems and Control Theory. SIAM, Philadelphia.

[3] Chadli, M., Daafouz, J., Darouach, M., 2008. Stabilisation of singular LPV systems, in: Proc. of the IFAC World Congress, pp. 9999-10002.

[4] Chadli, M., Darouach, M., 2011. Robust admissibility of uncertain swithced singular systems. International Journal of Control 84(10), 15871600.

[5] Cortes, J., 2008. Discontinuous dynamical systems, a tutorial on solutions, nonsmooth analysis, and stability. IEEE Control Systems Magazine 3, 36-73.

[6] Daafouz, J., Riedinger, P., Iung, C., 2002. Stability analysis and control synthesis for switched systems: A switched Lyapunov function approach, in: Proc. of the IFAC World Congress, pp. 1883-1887.

[7] Dai, L., 1989. Singular Control Systems. Springer.

[8] Farral, N.E., Mhaskar, P., Christofides, P.D., 2005. Output feedback control of switched nonlinear systems using multiple Lyapunov functions. System \& Control Letters 54, 1163-1182.

[9] Gao, Z., Ding, S., 2007. Actuator fault robust estimation and fault-tolerant control for a class of nonlinear descriptor systems. Automatica 43(5), 912-920.

[10] Haidar, A., Boukas, E., 2008. Robust stability criteria for markovian jump singular systems with time varying delays, in: Proc. of the IEEE Conference on Decision and Control, pp. 4657-4662.

[11] Johansson, M., Rantzer, A., 1998. Computation of piecewise quadratic Lyapunov functions for hybrid systems. IEEE Transactions on Automatic Control 43(4), 555-559.

[12] Koenig, D., 2005. Unknown input proportional multiple-integral observer design for linear descriptor systems: application to state and fault estimation. IEEE Transactions on Automatic Control 50(2), 212-217.

[13] Koenig, D., Marx, B., 2009. $H_{\infty}$-filtering and state feedback control for discrete-time switched descriptor systems. IET Control Theory and Applications 3(6), 661-670.

[14] Koenig, D., Marx, B., Jacquet, D., 2008. Unknown input observers for switched nonlinear discrete time descriptor systems. IEEE Transactions on Automatic Control 53(1), 373-379.

[15] Lieberzon, D., Morse, A.S., 1999. Basic problems in stability and design of switching system. IEEE Control Systems Magazine 19(5), 59-70.

[16] Luenberger, D., 1977. Dynamic equations in descriptor form. IEEE Transactions on Automatic Control 22, 312-320.

[17] Ma, S., Boukas, E., 2008. Stability and robust stabilization for uncertain discrete stochastic hybrid singular systems with time-delay, in: Proc. of the IEEE Conference on Decision and Control, pp. 3404-3409.

[18] Marx, B., Koenig, D., Georges, D., 2004. Robust fault tolerant control for descriptor systems. IEEE Transactions on Automatic Control 49, 18691875 .

[19] Masubuchi, I., Kamitane, Y., Ohara, A., Suda, N., 1997. $H_{\infty}$-control for descriptor systems: A matrix inequalities approach. Automatica 33(4), $669-673$. 

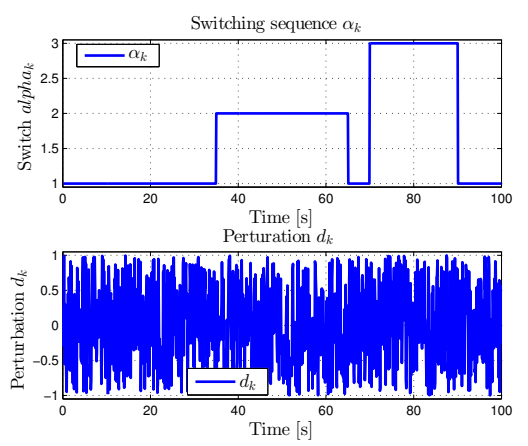

(a) Switching sequence $\alpha_{k}$ and disturbance $d_{k}$

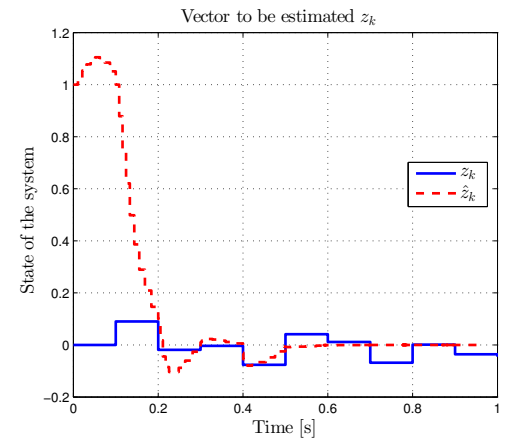

(d) $z_{k}:$ zoom on transient response
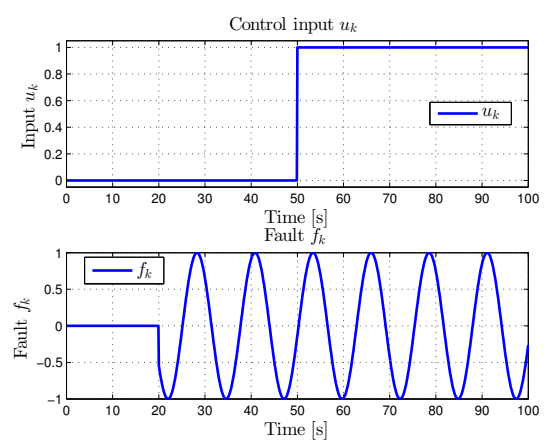

(b) Control input $u_{k}$ and fault $f_{k}$

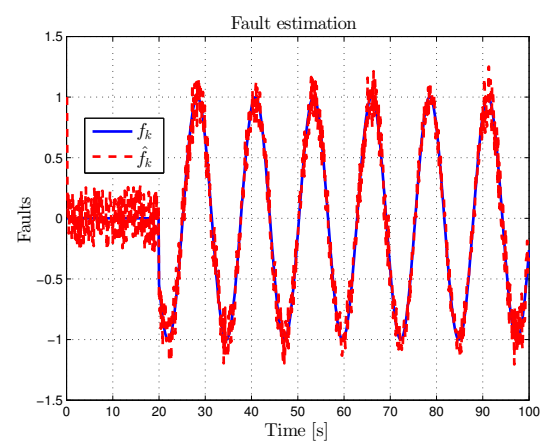

(e) Fault estimation without filters

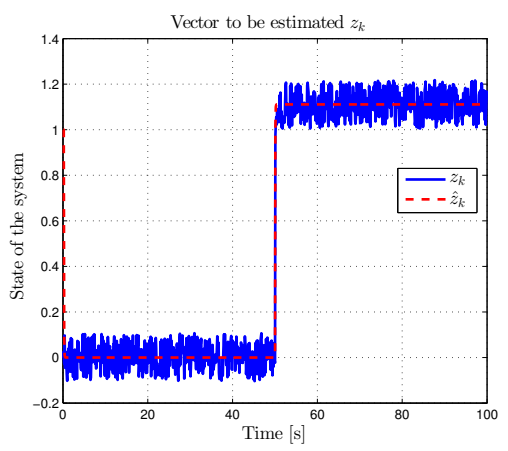

(c) $z_{k}$ and its estimate

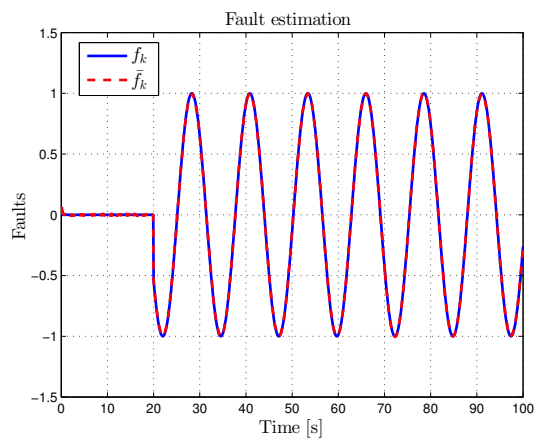

(f) Fault estimation with $W_{d}$ and $W_{f}$

Figure 3: Simulation Results

[20] Sun, Y.G., Wang, L., Xie, G., 2006. Delay-dependent robust stability and stabilization for discrete-time switched systems with mode-dependent time-varying delays. Applied mathematics and computation 180(2), 428435.

[21] Sun, Z., Ge, S., 2005. Analysis and synthesis of switched linear control systems. Automatica 41, 181-195.

[22] Xu, S., Lam, J., 2006. Robust Control and Filtering of singular Systems. Spinger.

[23] Xu, S., Yang, C., 1999. Stabilization of discrete-time singular systems: a matrix inequalities approach. Automatica 35, 1613-1617. 